\title{
Review of: "Distinct Roles of Dopamine D1 and D2 Receptor-expressing Neurons in the Nucleus Accumbens for a Strategy Dependent Decision Making"
}

\author{
Sho Yagishita
}

Potential competing interests: The author(s) declared that no potential competing interests exist.

In this paper, the authors manipulated and recorded the activity of D1 and D2 medium-spiny neurons (MSNs) in the nucleus accumbens of mice in behavioral tasks in which they had to either choose a cue that predicted a reward or avoid a cue that predicted no reward. The results showed that D2-MSN activity increased when the wrong choice was made and that this activity was necessary to make the right choice. Furthermore, the authors showed that the error responsiveness of D2-MSNs is acquired through a learning, as demonstrated by neuronal recordings over time. From these results, the authors conclude that D2 cell firing after an error correct the choice for the next trial. This conclusion generally supports previous findings by the authors and other groups. Furthermore, the finding of the activity patterns of D2-MSNs has implications for future research. Overall, the behavioral experiments were well designed, but I had several concerns in the analysis and interpretation of the experimental results.

\section{Major points}

1. The experiments were performed using a combination of D1-Cre/D2-Cre mice and AAVs containing promoters that are ubiquitously expressed. This approach has been reported to include cell types other than MSNs (e.g. Shin et al., Nat Comm 2018). On the other hand, the authors attribute all of their results to MSNs. Is this interpretation experimentally justified? In particular, this affects the interpretation of minority populations in the GCaMP analysis.

2. It is interesting that the suppression of D2-MSNs only during the outcome phase had a behavioral effect. To interpret the results I have a few concern. In Fig. 3 D-F, are these data analyzed with multiple comparisons? The author often states that the activity of D2-MSNs affects the next trial, but this claim needs to be confirmed by analyzing whether it affects only the next trial and not the one after it.

3. At the timing of cue presentation, inhibition of D2-MSNs has no effect and GCaMP imaging showed no difference after ROC processing when time locked with responses. It needs to be further confirmed that even if calcium imaging time locks on cue presentation, no activity is seen, and that inhibition of D2-MSNs during cue presentation has no effect on subsequent responses. 
4. If the firing of the D2-MSN is truly important only in the trial in which the error was made, and there is no effect during cue presentation, then the D2-MSN is not relevant in every correct choice. It may be inappropriate to interpret this result as "avoid a bad option under reward-based decision making". Also, can the authors deny the possibility of the increase in exploratory behavior after D2-MSNs inhibition?

\section{Minor points}

1. At I. 19, the authors state "the execution of the strategy to choose a good option or one to avoid a bad option under decision making". In the present behavioral experimental design, the former and the latter were conducted in different experiments, so it cannot be said that this strategy is mentioned in the present study.

2. Why does the percentage of Type I cells seem to be different between Fig. 4L and Fig. 5E Expert?

3. I.269-270 and I.319-I.323 seem to contradict each other. The latter argument seems to be new and important.

4. The discussion in I. 278-289 was not clear to me.

5. Please specify the promoter of GCaMP at I. 617. 